
stratégies de formation

\title{
De la mise en page à la mise en écran
}

Présentation de documents et de TD destinés à mettre en évidence les emprunts du J.T. (Journal Télévisé) à la P.E. (Presse Écrite)

\section{Odile Ausina}

\section{(2) OpenEdition}

\section{Journals}

Édition électronique

URL : http://journals.openedition.org/edc/2858

DOI : $10.4000 /$ edc.2858

ISSN : 2101-0366

Éditeur

Université Lille-3

Édition imprimée

Date de publication : 1 mai 1989

Pagination : $93-100$

ISSN : $1270-6841$

Référence électronique

Odile Ausina, «De la mise en page à la mise en écran », Études de communication [En ligne], 10 | 1989, mis en ligne le 09 juin 2013, consulté le 19 avril 2019. URL : http://journals.openedition.org/edc/2858 DOI : 10.4000/edc.2858

Ce document a été généré automatiquement le 19 avril 2019

(c) Tous droits réservés 


\section{De la mise en page à la mise en écran}

Présentation de documents et de TD destinés à mettre en évidence les emprunts du J.T. (Journal Télévisé) à la P.E. (Presse Écrite)

\section{Odile Ausina}

\section{INTRODUCTION.}

1 Les travaux dirigés commencent par une discussion autour des idées suivantes...

Chaque J.T. doit :

- mobiliser ses plus par rapport à d'autres médias et par rapport aux supports concurrents (course à l'audience),

- gommer ses handicaps par rapport à d'autres médias (moments imposés dans la journée, durées limitées, hiérarchie, concurrence, délais).

3 Selon les acquis antérieurs on développe quelques notions ; par exemple :

4 délais : désavantage en temps par rapport à la radio (mais avantage par rapport à la presse écrite),

5 concurrence:

- concurrence directe toujours plus importante vu la multiplication des chaînes qui favorise le zapping (48\% des foyers sont équipés de télécommandes),

- concurrence indirecte (magnétoscopes, hi-fi, radio, livres, conversation, autres...), « figures imposées du J.T. » : le voir à heures fixes, durée fixe (par exemple : impossible de raccourcir ou de développer un sujet sans amputer les autres), hiérarchisation fixe (chronologie).

Il faut donc amener le téléspectateur à accepter des contraintes :

- être au rendez-vous,

- rester « en chaîne » malgré le parcours imposé,

- enchaîner sur le programme qui suit (avec la pub qui l'enrobe)... 
7 Aussi bien l'enjeu est d'ordre économique, la manne publicitaire dépendant largement de l'audience.

8 A partir de cette discussion est posée la question de base :

Comment réussir ces figures imposées et garder le téléspectateur en chaîne ?

9 Une hypothèse de réponse est proposée aux étudiants :

Un travail qui porte largement sur la mise en forme et puise dans les techniques de mise en page de la presse écrite.

Des travaux dirigés vont permettre d'évaluer cette hypothèse...

\section{CONNAISSANCES PRÉALABLES.}

11 Pour ces TD on suppose deux séries de travaux, en amont :

- la confection du J.T. et ses contraintes,

- la presse écrite et le travail de maquette et de mise en page. On en fournit ci-après un bref conducteur donnant des références claires pour les TD présentés.

12 Nous avons pour notre part mené ces travaux avec des étudiants de première année. Mais ces travaux, de même que ceux qui font l'objet de cet atelier peuvent être à notre sens adaptés à des élèves du secondaire.

Conducteur P.E.

1. Introduction : Problèmes généraux de lectorat, concurrence, contexte économique, et donc nécessité de fidélisation.

2. Caractéristiques par rapport à d'autres médias et selon le type de supports (les plus, les moins) ; notamment, liberté pour le lecteur de circuler dans le support avec le rythme et les itinéraires qu'il veut (feuilleter, disposer de plusieurs niveaux de lecture) ou, en corollaire, de l'abandonner très vite et même de ne pas faire l'acte d'achat, de prise en main.

3. Souci P.E. de capter puis retenir l'attention du lecteur toujours prête à être distraite si le texte est long et/ou complexe et/ou peu accrocheur, etc...

- par le choix de sujets (en fonction du lectorat, de l'actualité, de la proximité, etc...),

- par le traitement du contenu (style, éclairage, quantité d'infos),

- par la maquette et la mise en page avec fonctions d'identification (originalité, esthétique), d'appel, de support de l'attention, de soutien de l'intérêt, etc...

Maquette et mise en page fournissent les références de l'atelier J.T. avec notamment les notions suivantes :

a) Impression Générale :

- format du journal, titre et son graphisme, couleur(s) d'accompagnement, colonnes, choix typographiques,

- physionomie de la Une (et secondairement de la dernière page) avec sommaire ou présommaire, éditorial, habitudes quant à l'organisation de la page, sujet(s) d'ouverture, nombres de sujets, photos avec ou sans légende, couleur ou noir et blanc, dessins, titraille, chapeaux, débuts d'articles et renvois en pages intérieures, articles complets, filets, bandeaux, etc...

b) Organisation :

- physionomie de l'ensemble du journal avec ses cahiers et/ou rubriques, leur choix et dénomination, leur importance relative (situation dans le journal, surface, régularité), 
- les procédés qui valorisent, articulent et balisent les différentes informations comme la titraille, le style et le corps des caractères, les schémas, graphismes, vignettes, photos (nombreuses, rares, détourées, habillées, etc...).

17 c) Approche comparée de différents supports ; la périodicité et la vocation des supports (quotidiens, magazines, nationaux, régionaux, généralistes, spécialisés, etc.) impliquent des styles et des choix de maquette différents.

d) Réflexion quant aux logiques sous-jacentes : contraintes liées au lectorat, aux éditions, à la fabrication et à la diffusion, à l'idéologie.

\section{TRAVAUX DIRIGÉS.}

19 Nous présentons d'abord les réflexions qui sous tendent les travaux dirigés et que l'on espère voir illustrer par ceux-ci. Nous proposons ensuite des modalités possibles de travail et quelques exemples de séquences avant de terminer par une présentation des objets de travail proposés dans le cadre de l'atelier en novembre dernier et par quelques remarques.

\section{Réflexions sur la mise en écran.}

C'est avant tout un travail de surface avec des facilitateurs de suivi: pour éviter le décrochage de l'attention, permettre de l'accrocher ou de la raccrocher en cours de route, le J.T. est accompagné de balises, clignotants, avertisseurs, décors, jeux de scène, gadgets. Ils sont destinés à donner du rythme, éviter l'uniformité, créer un spectacle avec multiplication des acteurs mais signature constante (les bandeaux de A2, son logo, sa star, ses couleurs).

Pour qu'il y ait rythme et spectacle dans un domaine où l'on est à la pointe de l'actualité... il faut mobiliser les moyens de la modernité et leurs gadgets techniques au look actuel, branché.

Mais derrière ces moyens sophistiqués, à variables désordonnées dans un contexte de lutte contre la concurrence, de moyens financiers disparates et de transformation du «genre J.T.» (apparition de formules plus courtes, disparition du présentateur, transformation $\mathrm{du}$ journaliste en speaker) ne retrouve-t-on pas la rhétorique plus traditionnelle de la presse écrite?

Il existe en fait un dénominateur commun au J.T. et à la P.E., handicap inévitable auquel il faut pallier; d'un côté il se manifeste sous forme de parole, celle du J.T., stéréotypée à force de vouloir concilier concision et simplicité, de l'autre, il se manifeste par le texte écrit et une compacité d'aspect que les journaux fuient par les ressources de la mise en page (cf. TD presse écrite) :

Dans les deux cas les médias se heurtent avec des modalités différentes à la loi du moindre effort. Pour le J.T. cette loi du moindre effort concerne le téléspectateur : la parole du J.T. est captée par une oreille susceptible d'en capter d'autres, de se laisser distraire,

- à moins que la parole du J.T. ne soit suffisamment «captivante ", intéressante, passionnante..., 
- à moins qu'à la parole du J.T. s'ajoutent des adjuvants qui donnent le change, participent à la captation, soutiennent l'attention... quitte à distraire de l'écoute active, mais en anesthésiant toute velléité de quitter la chaîne.

Parmi ces adjuvants, on retrouve dans la» mise en écran » les procédés de "mise en page ». Son étude peut se révéler intéressante à plusieurs égards ; élie peut notamment fournir :

- des angles fructueux pour un travail à visée directement pédagogique et de nombreuses possibilités de les adapter à des âges, contextes et préoccupations différents,

- une ouverture sur une autre réflexion: de la "mise en écran" au "show» (la nuit américaine des élections avec Christine Ockrent à New York en est un exemple),

- une recherche de modèles pour une utilisation performante de procédés qui, dans l'ensemble, apparaissent placés sous le signe de l'empirisme vu les enjeux et les contraintes. Cette recherche viserait par exemple à améliorer la qualité de l'information, à compenser les manques dus au temps imparti ou à la fugitivité de la parole, à imaginer pour le J.T. des règles fondées sur la complémentarité des différents supports du sens.

\section{Modalités de travail et séquences possibles.}

Travaux différents par petits groupes ou travaux communs successifs selon nombre, temps, corpus, acquis antérieurs. La présentation des unités de travail ci-après correspond à un souci de clarté et de diversification d'approches qui de fait entrent très vite en interférence.

A titre personnel, et avec des étudiants de 1ère année, nous avons eu l'occasion de traiter très rapidement la phase $\mathrm{A}$, compte tenu de travaux antérieurs sur la relation texte-image hors plateau dans les J.T. Nous avons par contre travaillé plus longuement sur la nature et le fonctionnement du texte écrit et des vignettes situées dans le coin supérieur droit ou gauche de l'écran.

\section{A. Images naturelles hors plateau.}

Nous regroupons ici des photos ou des films d'actualité et d'archives diffusés tels quels ou après montage.

Question 1.

Qu'aurait-on fait en P.E. à partir de ces images ?... Choix d'une photo ?... de plusieurs photos ?... En fonction de quoi ?... (La réflexion peut être modulée compte tenu de supports particuliers).

31 Objectif.

- Montrer que par rapport à la P.E. il existe rarement un plus réel quant à la valeur informative.

- En corollaire, montrer les caractéristiques qui permettent à une bonne photo de P.E. d'apporter des informations égales ou supérieures à une séquence du J.T.; prendre en compte l'opposition fugitivité/permanence.

Question 2.

Classification possible: images en direct, montage, archives?... Vidéo ou séquences d'images fixes ?... Degré d'originalité ? Évaluation (quantitative et qualitative) des sujets où l'image TV est « imbattable »...? 
Objectif.

- Montrer que peu d'images présentent le caractère d'actualité brûlante que l'on attribue souvent au J.T. et que le reportage avec images en direct est peu fréquent, lorsqu'il ne s'agit pas de rendre compte de rituels sociopolitiques ou d'événements créés de toutes pièces.

- Amener la notion de spectaculaire et montrer qu'un événement ne l'est pas par la simple vertu du direct. Montrer que le scoop image tient d'un hasard - indication « film d'amateur » (quelqu'un de non professionnel s'est trouvé là et a filmé ou photographié) - ou d'un demihasard - le décollage d'une fusée filmé en direct est banal; le spectaculaire vient de l'explosion et de la dimension humaine (équipage qui comprend des non spationautes).

- Montrer que l'intérêt des images du J.T. passe davantage par une valeur ajoutée (le discours du montage et son commentaire) que par le caractère récent des images.

Question 3.

Quelles sont les fonctions de ces images par rapport au reste de l'information (parole du présentateur, commentaire off, texte écrit, dessins, etc...)?

Objectif.

- Montrer que les fonctions d'ancrage et notamment d'identification l'emportent sur les fonctions de relais.

- Montrer que les images sont moins» informatives » qu'il n'y paraît, que l'on retrouve régulièrement des images leit-motives (Liban, lieux de grèves, Palestine, etc.) qui jouent au mieux un rôle «incitatif » (montrer les protagonistes d'un procès, la reconstitution d'un accident spectaculaire).

\section{Premier bilan : l'image en presse écrite et à la télévision.}

Comme en presse écrite l'image du journal, télévisé intervient largement comme un artifice de mise en page destiné à alléger une compacité de la parole comparable à la compacité de la copie-presse à l'état brut. Elle montre davantage ce dont on parle.

\section{B. Autres procédés de mise en écran.}

37 Question 1.

Inventaire.

- Forme: photo-montages, vignettes en incrustation ou surimpression, animations infographiques, textes, filets, bandeaux, etc., intégrés à la prise de vue plateau ou en relation au commentaire du présentateur principal ou de tel spécialiste. .

- Contenu : référents traditionnels ou pas.

- Emprunts à la P.E. directs ou modifiés.

Objectif.

- Montrer que le J.T. emprunte des techniques classiques de la presse écrite : pour le journal d'Antenne 2 par exemple, identification constante du support (couleurs, sigle), titrage et sur-titrage en ouverture du journal à la manière de la une en presse écrite, rubriquage par texte et vignettes dans le coin supérieur gauche de l'écran où l'on retrouve le balisage du journal papier, légendage d'jdentification de lieux et de personnes, etc.

- Mettre en évidence l'hétérogénéité des contenus (logos ou sigles en alternance avec des photos, désignation de rubriques mêlée à désignation de sujets, etc.). 
Question 2

Fonctions par rapport à l'ensemble des discours du J.T. et par rapport à la parole; repérage des sujets et rubriques par rapport à la P.E. ; les procédés de mise en écran les marquent-ils ?... de façon systématisée ou aléatoire ?... en empruntant à la P.E. dans quelle mesure?

Question 3.

Analyse sémiologique des vignettes (icônes, graphismes, teintes) avec notamment la question de leur intelligibilité, de la lisibilité des symboles ou des icônes figuratifs.

- Montrer que l'on retrouve ici le souci d'alléger l'information orale par des indications écrites, des marqueurs visuels ou autres procédés icôniques.

- Montrer que l'on trouve ici moins de régularité et d'homogénéité que dans la P.E. : mélange rubriques et sujets sous un aspect formel identique, hétérogénéité des référents mobilisés dans les vignettes. Ainsi les cartes USA et Soudan ne présentent probablement pas le même degré d'intelligibilité et donc de redondance avec le reste de l'information, leur utilisation répond probablement davantage à une facilité de réalisation qu'à un choix informatif; de même pour le mélange de photos et de logos avec, à l'intérieur des séries, des degrés divers de familiarité et de fonctionnement de la référenciation (emblèmes de l'Université de Bologne, choix d'un train détourné qui devient le marqueur des épisodes successifs de l'affaire de l'accident du Luxembourg-Paris).

\section{Second bilan : réflexion sur le rôle des procédés de mise en écran.}

Ces procédés vont-ils au-delà de ceux que la mise en page utilise ? Pourrait-on, à ce niveau, imaginer une " grammaire de l'événement » ${ }^{1}$ ?

\section{Les documents de travail présentés en atelier.}

43 Nous avions prévu deux types de documents et, tout d'abord, l'enregistrement d'un J.T. d'A2 à 20 heures à comparer avec un J.T. de TF1 même jour, même heure.

Nous voulions ainsi montrer des différences qualitatives et quantitatives dans les procédés de mise en écran; actuellement A2 les mobilise bien plus que TF1 et de façon très systématisée.

Nous n'avons pas eu le temps de mener une comparaison ; nous avons simplement essayé de montrer par la diffusion du J.T. de la deuxième chaîne l'importance et la variété des procédés.

ous avons, par ailleurs, présenté un montage bout à bout des vignettes plateau situées en haut de l'écran pour amorcer une classification possible et un questionnement sur la nature et le rôle de ces vignettes.

47 En fait, et compte tenu du temps très lourd occupé par la diffusion, le débat a surtout porté sur des problèmes de terminologie qui, de mon point de vue, n'exigent pas d'être résolus avant de pouvoir travailler de manière satisfaisante. 

l'écran, il me semble que le fait intéressant reste que le second se comporte comme un «à plat ». soient situées en haut, à droite ou à gauche de l'écran (le choix a bien sûr des conséquences sur leur fonctionnement) ; cette situation, les contenus et leur mise en forme comptent davantage qu'une dénomination que les ressources techniques de la modernité peuvent mettre à mal à court terme.

Reste que régler des aspects terminologiques et arrêter par exemple ce que l'on peut légitimement classer sous le mot» vignette » peut simplifier les choses. A condition de ne pas vouloir absolument trouver un ordre là où il y a désordre d'un genre journalistique en pleine remise en question.

\section{BIBLIOGRAPHIE}

Hermelin, Presse Actualités, Bayard, nº 172-176.

\section{NOTES}

1. Hermelin, Presse Actualités, Bayard, n 172-176.

\section{RÉSUMÉS}

Autour d'une réflexion, menée en travaux dirigés, sur le journal télévision l'auteur a mis en place des exercices consistant à réaliser un journal télévisé en prenant en compte ses caractéristiques, ses contraintes, ses concurrents (radio), etc. Ces exercices ont pour but de répondre à la question de savoir comment garder le téléspectateur devant l'écran. Ils passent notamment par une réflexion sur la mise en forme, en partie inspirée de la mise en page dans la presse écrite.

\section{INDEX}

Mots-clés : journal télévisé, presse écrite, mise en page, visualisation d'information

Keywords : television news, print media, layout, information visualization 
AUTEUR

ODILE AUSINA

Odile Ausina, Université de Lille III 